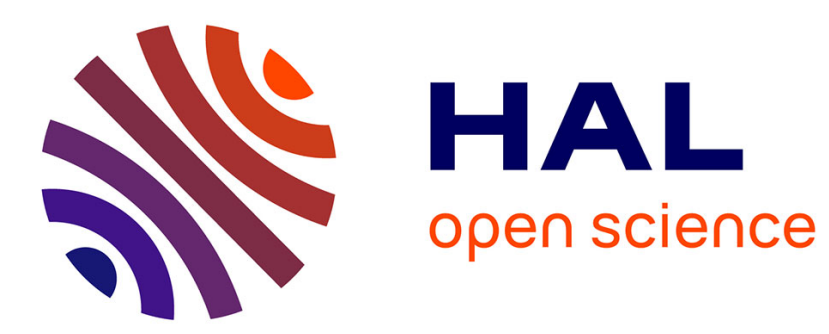

\title{
Improving ECG beats delineation with an evolutionary optimization process.
}

\author{
Jérôme Dumont, Alfredo I. Hernandez, Guy Carrault
}

\section{To cite this version:}

Jérôme Dumont, Alfredo I. Hernandez, Guy Carrault. Improving ECG beats delineation with an evolutionary optimization process.. IEEE Transactions on Biomedical Engineering, 2010, 57 (3), pp.607-15. 10.1109/TBME.2008.2002157 . inserm-00361377

\section{HAL Id: inserm-00361377 https://www.hal.inserm.fr/inserm-00361377}

Submitted on 26 May 2009

HAL is a multi-disciplinary open access archive for the deposit and dissemination of scientific research documents, whether they are published or not. The documents may come from teaching and research institutions in France or abroad, or from public or private research centers.
L'archive ouverte pluridisciplinaire HAL, est destinée au dépôt et à la diffusion de documents scientifiques de niveau recherche, publiés ou non, émanant des établissements d'enseignement et de recherche français ou étrangers, des laboratoires publics ou privés. 


\title{
Improving ECG Beats Delineation with an Evolutionary Optimization Process
}

\author{
J. Dumont, A.I. Hernández, G. Carrault \\ INSERM, U642, Rennes, F-35000, France; \\ Université de Rennes 1, LTSI, Rennes, F-35000, France; \\ LTSI, Campus de Beaulieu, Université de Rennes 1, \\ 263 Avenue du General Leclerc - CS 74205 - 35042 Rennes Cedex, France
}

\begin{abstract}
As in other complex signal processing tasks, ECG beat delineation algorithms are usually constituted of a set of processing modules, each one characterized by a certain number of parameters (filter cutoff frequencies, threshold levels, time windows....). It is well recognized that the adjustment of these parameters is a complex task that is traditionally performed empirically and manually, based on the experience of the designer. In this work, we propose a new automated and quantitative method to optimize the parameters of such complex signal processing algorithms. To solve this multiobjective optimization problem, an evolutionary algorithm (EA) is proposed. This method for parameter optimization is applied to a Wavelet-Transform-based ECG delineator that has previously shown to present interesting performances. An evaluation of the final delineator, using the optimal parameters, has been performed on the QT database from Physionet and results are compared with previous algorithms reported in the literature. The optimized parameters provide a more accurate delineation, with a global improvement, over all the criteria evaluated, and over the best results find in the literature, measured of $7.7 \%$, which is a proof of the interest of the approach.
\end{abstract}

Index Terms-Electrocardiography, Wavelet transforms, Optimization methods, Genetic algorithms

\section{INTRODUCTION}

ECG wave delineators supply fundamental features, like peak amplitudes (peaks of $\mathrm{P}, \mathrm{T}$ or $\mathrm{R}$ waves) and wave intervals (PR, QT...) for each detected beat. These features can be used to formulate hypotheses on the underlying physiological phenomena or as a previous step in automatic ECG analysis systems. Performing an accurate segmentation is not an easy task and results obtained with current algorithms are not always satisfactory. A recurrent problem encountered in these algorithms is the adjustment of the numerous parameters. For example, several Wavelet-Transform (WT) based algorithms have already been proposed in the literature to perform a segmentation of ECG beats [1], [2], [3], [4], [5]. They have demonstrated good performances compared to other methods such as low pass differentiators [6], mathematical models [7], adaptive filtering [8] or dynamic time warping [9] and thus have been retained for our purpose. Although this method is globally appropriate to this segmentation task, it presents some limitations: firstly, it requires the definition of a great number

This work has been partly supported by the ECOS-NORD cooperation program, action number V03S03. of parameters (thresholds, time windows...), secondly, there is no obvious way to tune all these parameters in a joint manner, mostly because the detection is performed in the time-scale domain and a priori physiological information, like potential wave position, is harder to use. In previous approaches, all these parameters are usually defined empirically and manually and, considering the complexity of the problem (great number of beat morphologies, no universal definition of the boundary positions...), it is clear that the delineation can be improved if the parameters are optimized by a rigorous process.

To achieve this goal, in the present work, an optimization methodology based on evolutionary algorithms has been designed to tune the parameters of an ECG WT-based delineator. However, this methodology is generic and it could be easily transposed to any kind of delineators, detectors or classifiers that are characterized by many parameters.

The remainder of this paper is organized as follows. Section II gives an overview of the methodology, presenting the delineation process (subsection II-A) and the optimization process (subsection II-B). Section III presents the results in two parts: firstly, in subsection III-A, the learning stage is described and the parameters obtained are commented. Secondly, in subsection III-B, the delineator has been evaluated on an annotated database and with the optimal parameters. The performances are compared to those published in the literature.

\section{Proposed Methodology}

The objective here is to adjust the parameters of an ECG delineator in order to get similar results between the automatic detector and manual annotations, stored by cardiologists in biosignal databases, and supposed here as the reference. The whole procedure is depicted in Fig. 1. It involves three important components: i) the annotated database, ii) the segmentation algorithm and its parameters iii) the optimization method, which includes a procedure to adjust the parameters of the delineator and the definition of a cost function, to be minimized. As already mentioned, the proposed methodology is generic and can be used for other complex signal-processing algorithms. However, in this paper, we will present a particular application to WT-based ECG delineators. The two following subsections detail, firstly, the delineator algorithm and secondly, the cost function and the optimization method. 


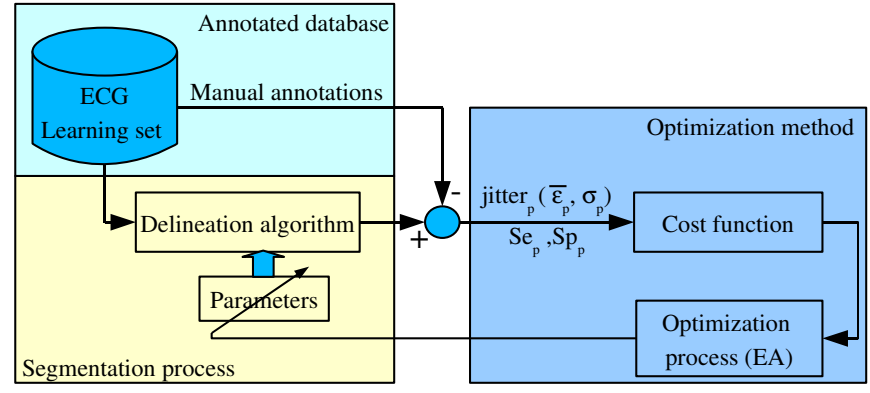

Fig. 1. Schematic representation of the proposed optimization process for tuning the delineator parameters (see text for further details).

\section{A. Segmentation algorithm}

Performing a reliable detection of the ECG wave boundaries, as illustrated figure $2 \mathrm{~d}$ ), is a difficult task for several reasons: i) the ECG can have a low signal-to-noise ratio, ii) a great variety of wave morphologies exists, even across healthy subjects, iii) there is no universal definition of where the wave boundaries are located (in particular for the Q-wave).

Multiscale approaches, which permit noise reduction at rough scales and then to refine the position with the help of finer scales, provide a robust detection procedure compared to other methods.

In this work, the wave positions are computed through a three-stage process, presented in Fig. 2.

Algorithms taken from the literature were used for the first two stages: baseline drift cancellation [10] and QRS detection [11].

The third stage, inspired from the work of Martinez et al. [2], applies a dyadic WT decomposition and uses the different scales to find wave boundaries. The implementation of the dyadic decomposition is performed with a filter bank inspired from the "algorithme à trous" of Holschneider [12] and presented Fig. 3. Compared to the original filter bank decomposition, this algorithm allows to conserve the original time scale with only a dyadic scale for the levels. In this algorithm, the decomposition with a wavelet at scale $i$ $\left(\psi_{2^{i}}(t)=\frac{1}{\sqrt{2^{i}}} \psi\left(\frac{-t}{2^{i}}\right)\right)$ is implemented with a combination of two discrete filters, the approximation and the detail filters, with coefficients respectively noted $l[n]$ and $h[n]$. Taking into account that a filter $x^{j}[n]$ is the equivalent of a filter $x[n]$ with the insertion of $2^{(j-1)}$ zeros between each coefficient and that $\bar{x}[n]=x[-n]$, then the filters $a_{i}[n]$ and $d_{i}[n]$, producing the approximation and detail at level $i$, directly from the original signal, are given by:

$$
\begin{gathered}
a_{1}[n]=\bar{l}[n] \text { and } d_{1}[n]=h[n] \\
a_{j+1}[n]=a_{j} \star \bar{l}^{j}[n] \text { and } d_{j+1}[n]=a_{j} \star \bar{h}^{j}[n] \text { for } j \geq 1
\end{gathered}
$$

In our case, each beat is extracted from the ECG signal and delimited into a small temporal support to form the initial signal $W 2^{0}$ to be decomposed. During the decomposition only the detail levels of $W 2^{0}$ are retained for the segmentation:

$$
W 2^{j}=W 2^{0} \star d_{j}
$$

The mother wavelet $\psi(t)$, from which are derived the

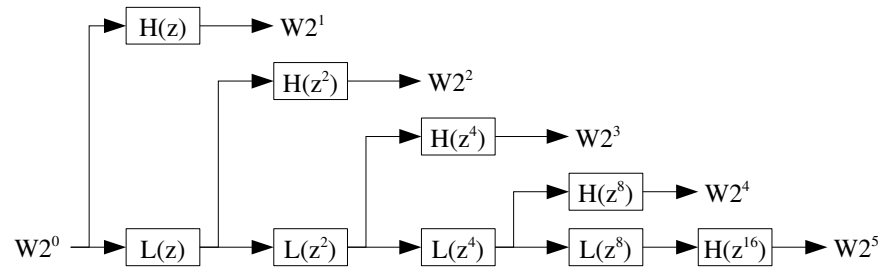

Fig. 3. Filter bank, $\mathrm{W} 2^{k}$ are the output of the filter at scales $2^{k}(\mathrm{k}=1$ to $5), \mathrm{W} 2^{0}$ is the original beat.

approximation filter $l[n]$ and the detail filter $h[n]$ is a quadratic spline and its Fourier transform is given by:

$$
\psi(\omega)=j \omega\left(\frac{\sin \left(\frac{\omega}{4}\right)}{\frac{\omega}{4}}\right)^{4}
$$

This wavelet can be seen as the derivative of a low-pass function. It is thus very useful to analyse the slopes of the different ECG waves and has already been successfully applied in this context [1], [5], [2].

Once the decomposition is performed, first the $\mathrm{R}$ wave, then the $\mathrm{Q}$ and $\mathrm{S}$ waves, and finally the $\mathrm{P}$ and $\mathrm{T}$ waves are respectively retrieved. The global procedure is as follows:

- The $\mathrm{R}$ wave is detected by analysing $W 2^{2}$, by looking for the two largest peaks of opposite sign, then by searching the zero-crossing on $W 2^{1}$ between these slopes. If several zero crossings appear, the one associated with the highest magnitude on $W 2^{0}$ is retained. Parameters R1 and R2 define the temporal support for the $\mathrm{R}$ wave search.

- The iso-electric level is determined with the method proposed by Smrdel and Jager [13].

- The Q and S waves, as well as QRSon and QRSoff, are retrieved by analysing the local maxima on the two first scales, $W 2^{1}$ and $W 2^{2}$, which have a quite high frequency content, as in Martinez [2]. The thresholds $\gamma_{Q R S p r e, Q R S p o s t}$ are proportional to the maximum slope of the $\mathrm{R}$ wave and are used to identify the other significant slopes of the $\mathrm{Q}$ and $\mathrm{S}$ waves. The temporal supports for searching these waves are defined by the parameters $Q R S_{\text {Qlim }, \text { Slim }}$.

- The $\mathrm{P}$ and $\mathrm{T}$ waves are delimited by analysing scales $W 2^{4}$ or $W 2^{5}$ : if these waves are not found at $W 2^{4}$, they are also searched at $W 2^{5}$. Their peaks are found on the immediate lower scale (i.e. $W 2^{3}$ or $W 2^{4}$ ). The threshold for waves detection are $\epsilon_{(P, T)}$ and are relative to the power at scales $W 2^{4}$ or $W 2^{5}$. The significant slopes are identified with respect to thresholds $\gamma_{T, P}$. Time windows for $\mathrm{P}$-wave and $\mathrm{T}$-wave detection depend on the previous RR interval (thresholds $\mathrm{P} \_\mathrm{RR}$ and $\mathrm{T} \_\mathrm{RR}$ ). T1 $\bullet / \mathrm{P} 1 \bullet$ are the left bounds of these windows and $\mathrm{T} 2 \bullet / \mathrm{P} 2 \bullet$ are the right bounds, $\bullet$ is the index that changes in function of the RR interval. The following rules, inspired from the work of Laguna [14] have been used:

- The wave onset and offset (for S, Q, P, and T waves) are detected with respect to the thresholds $\xi_{(Q R S o n, Q R S e n d, T o n, T e n d, P o n, P e n d)}$ relative to the amplitude of the first or last significant slope.

- Compared to the work of Martinez [2], an additional veri- 
a) ECG with baseline drift

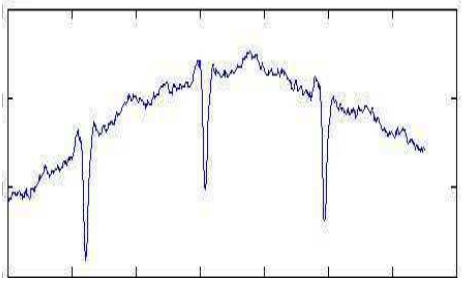

d) Beats are segmented

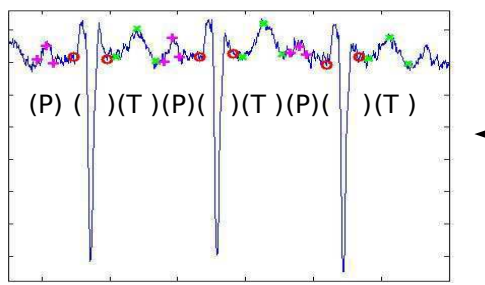

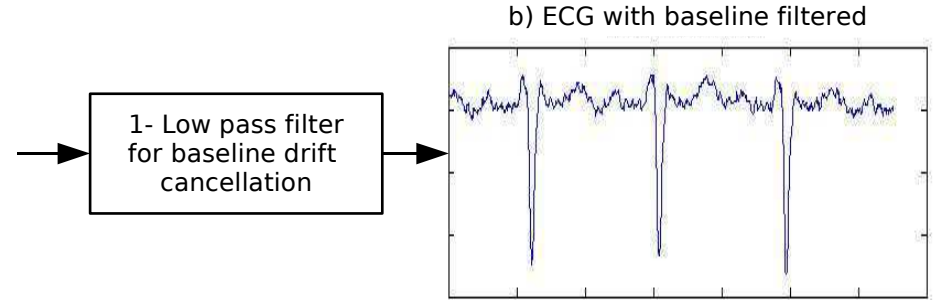

c) Beats are detected

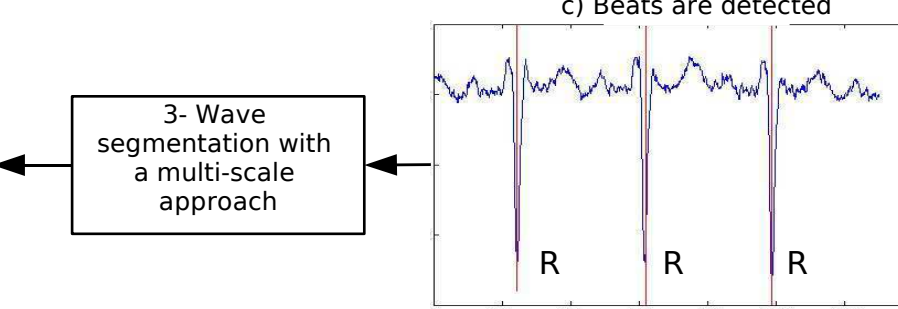

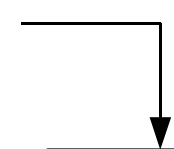

2- QRS detector: Pan \& Tompkins

Fig. 2. The three stages of the ECG wave segmentation process. The segmentation of each wave is graphically represented in d) using the following notations: (p) for the P wave onset (Pon), peak (Ppeak) and offset (Poff); () for the begining and end of the QRS (QRSon and QRSoff); (T) for the T wave onset (Ton), peak (Tpeak) and offset (Toff).
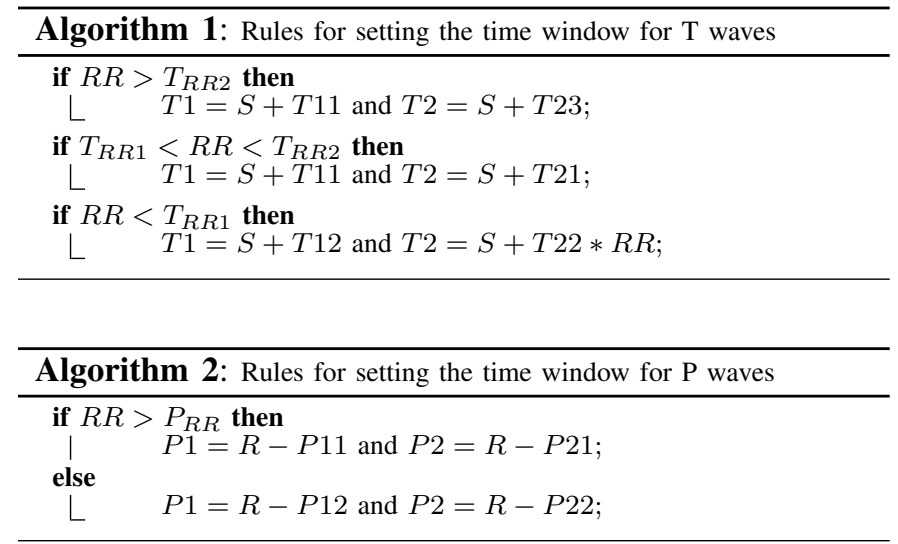

fication step is introduced to evaluate the segmentation of the $\mathrm{P}$ and $\mathrm{T}$ waves. Experiments show, for example, that offsets of the $\mathrm{T}$ waves can be detected on some peaks of the $\mathrm{P}$ waves, especially in the case of a high heart rate. The algorithm then commits an error when classifying this kind of $\mathrm{T}$ waves as biphasic (see section III for a quantitative evaluation of this error). To avoid such a problem, this verification step checks if the magnitude of the $\mathrm{P}$ or $\mathrm{T}$ waves are further away from the iso-electric level than their own onset and offset levels. If not, it is considered that the current delineation is wrong and the temporal support is redefined. This verification step is applied repeatedly, starting from a large temporal support and reducing it.

All the parameters described here are summarized in table II.

The segmentation of a $\mathrm{T}$ wave on a sinus beat and using scales 0,3 and 4 is illustrated in Fig. 4, as an example. The first plot presents the original beat $\left(W 2^{0}\right)$, the second is the decomposition at level 3 and the two last plots are decompositions at level four, with different temporal supports. The vertical lines are the boundaries of the temporal support.

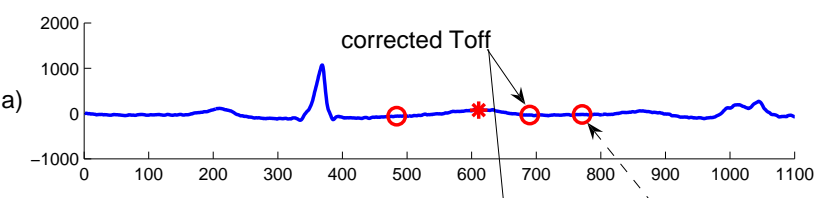

b)
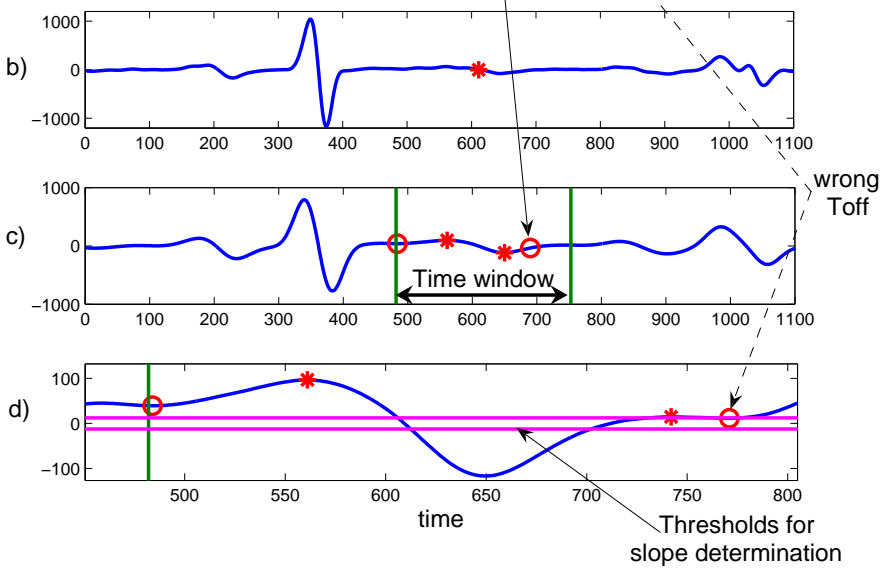

Fig. 4. $\mathrm{T}$ wave detection on a sinus beat from the QT database. The upper panel a) presents the original beat, $\left(W 2^{0}\right)$, the second panel b) is the decomposition at level 3 (W2 ${ }^{3}$ ), and the two last plots, c) and d) are the decompositions at level four (W2 ${ }^{4}$ ), with different temporal supports.

In the last subplot, a small maximum (the star on the right) is detected in this temporal window and just above the decision threshold for slope identification. Taking into account this maximum leads to a Toff position (extreme right circle on $W 2^{0}$ ) which does not correspond to the end of the $\mathrm{T}$ wave. The verification step identifies this problem and readjusts the temporal support so that this maximum is no longer taken into account. The final detected boundaries are presented on $W 2^{0}$ and correspond well to the annotations.

The performance of this approach depends naturally on the good definition of the analysing time windows, but also on the decision thresholds that specify which slopes are significant and those that define wave boundaries. Performance 
improvements with respect to previous works [1], [2], can thus be expected, by optimizing these parameters. The next section describes the proposed optimization method.

\section{B. Optimization process}

The aim of this process is to adjust all the parameters of the delineator, in order to minimize a cost function that describes its quality. The optimization procedure does not use any $a$ priori knowledge other than the manual annotations and is able to find a global solution in a high-dimensional search space. Its two main constituents, the cost function and the optimization algorithm itself, are detailed hereafter:

1) Cost function: It permits to obtain a quantitative description of the performance of the delineator, given a specific set of parameters. It combines several criteria:

- The detection error probability, defined here as:

$$
\operatorname{Perr}_{p}=\sqrt{\left(1-S e_{p}\right)^{2}+\left(1-S p_{p}\right)^{2}}
$$

where $S e_{p}$ and $S p_{p}$ are, respectively, the sensibility and the specificity obtained from the detector for indicator $p$ $(p \in$ Pon, Ppeak,... Tpeak, Toff $)$. It is computed as the distance between the point $\left(S e_{p}, S p_{p}\right)$ and the perfect detection point $(S e=1$ and $S p=1)$.

- The mean segmentation jitter $\overline{\varepsilon_{p}}$, which is the average jitter committed over all $M$ records and all $N_{m}$ beats of each record where indicator $p$ is detected and its annotations are also available:

$$
\overline{\varepsilon_{p}}=\frac{\sum_{m=1}^{M} \sum_{n=1}^{N_{m}} \text { annotated }_{p}(m, n)-\text { detected }_{p}(m, n)}{\sum_{m=1}^{M} N_{m}}
$$

- The standard deviation of the segmentation jitter $\sigma_{p}$. It is calculated as the mean of the standard deviation of the detection jitter for each record $\left(\sigma_{p}^{m}\right)$ which are weighted according to their number of beats:

$$
\sigma_{p}=\frac{\sum_{m}\left(N_{m} \sigma_{p}^{m}\right)}{\sum_{m} N_{m}}
$$

These three criteria are evaluated for all the characteristic points $p$. This kind of multi-objective optimization can be approached as a single-objective problem, with a cost function defined as a weighted average of the different criteria:

$$
O=\sum_{p=1}^{P}\left(\frac{\operatorname{Perr}_{p}}{a_{p}}+\frac{\overline{\varepsilon_{p}}}{b_{p}}+\frac{\sigma_{p}}{c_{p}}\right)
$$

Qualitatively, this cost function evaluates the improvement ratio between our results $\left(\operatorname{Perr}_{p}, \overline{\varepsilon_{p}}, \sigma_{p}\right)$ and the corresponding best results, $a_{p}, b_{p}$, and $c_{p}$, reported in the literature from [2] and [6] and for the three criteria. These parameters $a_{p}, b_{p}$, and $c_{p}$ are resumed in table I. It is the result of this cost function that is minimized by the optimization algorithm.

\begin{tabular}{|c|c|c|c|c|c|c|c|}
\hline & Pon & Ppeak & Poff & QRSon & QRSoff & Tpeak & Toff \\
\hline a (Perr) & 0.089 & 0.089 & 0.089 & 0.07 & 0.07 & 0.022 & 0.022 \\
\hline b $(\bar{\varepsilon})$ & 2.7 & 3.6 & 0.1 & 3.6 & 0.8 & 0.2 & 1.6 \\
\hline c $(\sigma)$ & 13.3 & 10.6 & 12.3 & 7.7 & 8.3 & 13.9 & 18.1 \\
\hline
\end{tabular}

TABLE I

REFERENCE CRITERIA ISSUED FROM THE BEST RESULTS PUBLISHED IN THE LITERATURE.

2) Optimization algorithm: It is based on an evolutionary algorithm. Standard gradient-based optimization methods cannot be used because the detector contains many threshold comparisons and thus the cost surface is discontinuous. On the other hand, stochastic search methods, such as evolutionary algorithms (EA), are particularly well adapted to this problem.

EAs are optimization techniques, inspired on the theories of evolution and natural selection, which can be employed to find an optimal configuration for a given system within specific constraints [15]. In these algorithms, each individual of a population is characterized by a set of parameters to be optimized (or chromosome). An initial population is created, usually from a set of random chromosomes, and this population will evolve, improving its global performance, by means of an iterative process. During this process, each individual is evaluated with a cost function, representing how its chromosome configuration can be a good solution for the given problem. A new generation is produced by applying mutation and crossover operators, with probabilities $p_{m}$ and $p_{c}$ respectively, on selected individuals presenting low values on their cost functions. EA have been applied in several biomedical applications to estimate a large set of parameters and have provided quite good results [16], [17]. The properties of EA for multimodal optimization has already been exploited in the context of features inspection for classification tasks [18]. Readers who are interested in more details concerning the implementation of EAs can refer to [15].

The optimization process adjusts the complete set of parameters. Knowledge on the segmentation algorithm have been introduced to facilitate this task, by taking into account that some parameters have to be jointly optimized (for example thresholds and windows for a specific wave), whereas others can be adjusted sequentially (the parameters concerning two different waves). This segmentation algorithm identifies firstly the $\mathrm{Q}, \mathrm{R}$ and $\mathrm{S}$ waves, secondly the $\mathrm{P}$ wave and thirdly the $\mathrm{T}$ wave. The detection of the $\mathrm{T}$ wave is based on a time window whose localisation is relative to the $S$ wave. Thus, finding the optimal window is only possible if the $S$ wave is optimally detected. In this direction, the optimization process is decomposed into two steps:

- In the first step, called EA1, the parameters playing a part in the detection of Pon, Peak, Poff, QRSon, and QRSoff are jointly optimized. Once this step is performed, the $S$ wave can be accurately defined.

- In a second step, EA2, parameters for Tpeak and Toff are optimized with the others parameters extracted from the best individual proposed at the last generation of EA1.

The interest of such a partitioning is to reduce the dimension of the search space and also the computation time. Specific 
details on the configuration of these two EA-based process are the following:

- individual coding : individuals are coded with realvalued chromosomes. Values for each parameter were bounded to a meaningful interval: time windows are defined from possible extreme positions and durations of each wave whereas boundaries of other thresholds are determined by largely increasing (upper bounds) and decreasing (lower bounds) parameters from [2]. These intervals are employed by the EA during the construction of the initial population.

- selection method and genetic operators: the ranking selection method was used in this work. Standard genetic operators for real-valued chromosomes were used: arithmetic and heuristic crossover, uniform mutation [15].

For these two steps, the population is trained over 80 generations and with 60 individuals. The probability of crossover, $p_{c}$, is set to 0.7 [19]. In order to obtain more reliable and stable solutions, the probability of mutation, $p_{m}$, has been adapted through the evolutionary process, starting at a high value during the first generations, to ensure a wider individual distribution over the search space and decreasing until the end, to facilitate the convergence to a reliable minimum. The solution of Back [20] has been retained according to the satisfying results which have been reported in the literature:

$$
p_{m}=\left(2+\frac{\left(N_{p}-2\right)}{\text { Maxgen }-1} * \text { gen }\right)^{-1}
$$

where $N_{p}$ is the number of parameters, Maxgen is the maximum number of generations, gen is the number of the current generation.

\section{RESULTS}

Results are presented in two parts. Firstly, an analysis of the parameters obtained after the learning phase is performed. Secondly, the delineation performance, obtained with the optimal parameters, are presented and compared to other results from the literature. The database used for this application is the QTDB from physionet [21]. The QTDB provides a wide variety of pathologies in a total of 105 records, with two channels sampled at $250 \mathrm{~Hz}$. Compared to other databases, it contains also a large number of annotated beats per record: 30 beats instead of 1 in the CSE database. The annotations are also very complete with all the positions of the onset, peak and offset points of the P, QRS and T waves. For all these reasons, this database has been used to train and to validate our delineator algorithm.

\section{A. Learning stage and optimal parameters}

Previous works on wavelet-based ECG segmentation rely on a manual definition of the set of parameters of the signal processing chain. In Martinez [2], a specific database is used to set the parameters but these last ones are empirically defined, as in the work of Li [1]. Considering the problems due to the number of morphologies, the number of parameters, and the competitive objectives, it is difficult to obtain reliable results from such an approach. Using the previously described

\begin{tabular}{|c|c|c|c|c|c|}
\hline \multicolumn{4}{|c|}{ EA1 } & \multicolumn{2}{|c|}{ EA2 } \\
\hline P11 & $278 \pm 31$ & $\gamma_{Q R S p r e}$ & $0.09 \pm 0.03$ & $\epsilon_{T}$ & $0.24 \pm 0.06$ \\
\hline P12 & $240 \pm 17$ & $\gamma_{Q R S p o s t}$ & $0.11 \pm 0.03$ & $\gamma_{T}$ & $0.28 \pm 0.07$ \\
\hline $\mathrm{P} 21$ & $88 \pm 14$ & $\xi_{Q R S o n p o s}$ & $0.07 \pm 0.04$ & $\xi_{\text {Ton }}$ & $0.17 \pm 0.09$ \\
\hline $\mathrm{P} 22$ & $99 \pm 27$ & $\xi_{Q R S o n n e g}$ & $0.07 \pm 0.04$ & $\xi_{\text {Tend }}$ & $0.36 \pm 0.07$ \\
\hline R1 & $118 \pm 34$ & $\xi_{Q R S e n d p o s}$ & $0.21 \pm 0.12$ & T11 & $111 \pm 24$ \\
\hline $\mathrm{R} 2$ & $111 \pm 37$ & $\xi_{Q R S e n d n e g}$ & $0.23 \pm 0.11$ & $\mathrm{~T} 21$ & $441 \pm 75$ \\
\hline$P_{R R}$ & $664 \pm 182$ & $Q R S_{\text {Qlim }}$ & $88 \pm 22$ & T12 & $90 \pm 16$ \\
\hline$\epsilon_{P}$ & $0.12 \pm 0.05$ & $Q R S_{\text {Slim }}$ & $154 \pm 32$ & T22 & $0.6 \pm 0.08$ \\
\hline$\gamma_{P}$ & $0.4 \pm 0.09$ & & & $\mathrm{~T} 23$ & $581 \pm 94$ \\
\hline$\xi_{P o n}$ & $0.41 \pm 0.08$ & & & $T_{R R 1}$ & $705 \pm 155$ \\
\hline$\xi_{\text {Pend }}$ & $0.76 \pm 0.05$ & & & $T_{R R 2}$ & $1231 \pm 70$ \\
\hline
\end{tabular}

TABLE II

PARAMETERS USED BY THE DELINEATOR (SUBSECTION II-A) WITH THEIR OPTIMAL VALUES, REPRESENTED AS MEAN \pm STANDARD DEVIATION, OBTAINED FROM THE OPTIMIZATION PROCESS.

optimization process on a manually annotated database, like the QTDB, can solve this problem.

To compare our results with those obtained with other methods [2], [21], it is required to perform a test on all the beats of the database. Since the training stage is also performed on the same database, a training set $L$ and test $T$ set have to be defined. To achieve this, all the records are firstly divided into three equivalent parts, called subrecords. Two thirds of all subrecords are randomly chosen from all subrecords and affected to a training set. The remaining subrecords are allocated to the test set. 13 couples of training/test sets are generated with this method in order to perform a cross-validation analysis of the performances of the delineator and to evaluate the sensitivity of the optimal parameters obtained to a particular instance of $L$ and $T . \mathrm{L}\{1-13\}$ and $\mathrm{T}\{1-13\}$ are respectively the training and test sets whereas $O P_{L\{1-13\}}$ are the optimized parameters.

An overview of all the parameters, optimized with the EA process carried out on the different learning sets, is presented in table II, with their means and standard deviations for the 13 different training sets.

The values of some of these optimal parameters present low variations, like the temporal windows for the $\mathrm{P}, \mathrm{Q}, \mathrm{S}$ and $\mathrm{T}$ waves or the thresholds that define the onset or offset of $\mathrm{P}$ and $\mathrm{T}$ waves, indicating the high sensitivity of the segmentation algorithm to these parameters. In the other hand, the temporal windows used to search the $\mathrm{R}$ wave or the thresholds that define the QRSonset and offset show a wider dispersion across the different learning sets. These results were expected and show the reduced sensitivity of the QRS segmentation step to these parameters. Indeed, the $\mathrm{R}$ wave presents commonly a high signal-to-noise ratio and is always very close to the fiducial point, the slopes of the QRS onset and offset are also more enhanced than the slopes of the $\mathrm{P}$ and $\mathrm{T}$ waves, so a wide range of parameters give approximately the same results. When only one set of parameters is required for future segmentations, the mean value of the parameters obtained over the different training sets, which can be expected to give the minimum of the cost function, found on the QTDB, is conserved. It is important to underline that the proposed approach is not only particularly useful for the tuning of these parameters, but also to analyse the sensitivity of each parameter with respect to the final detection results.

The convergence of the EA is also verified, specially accord- 


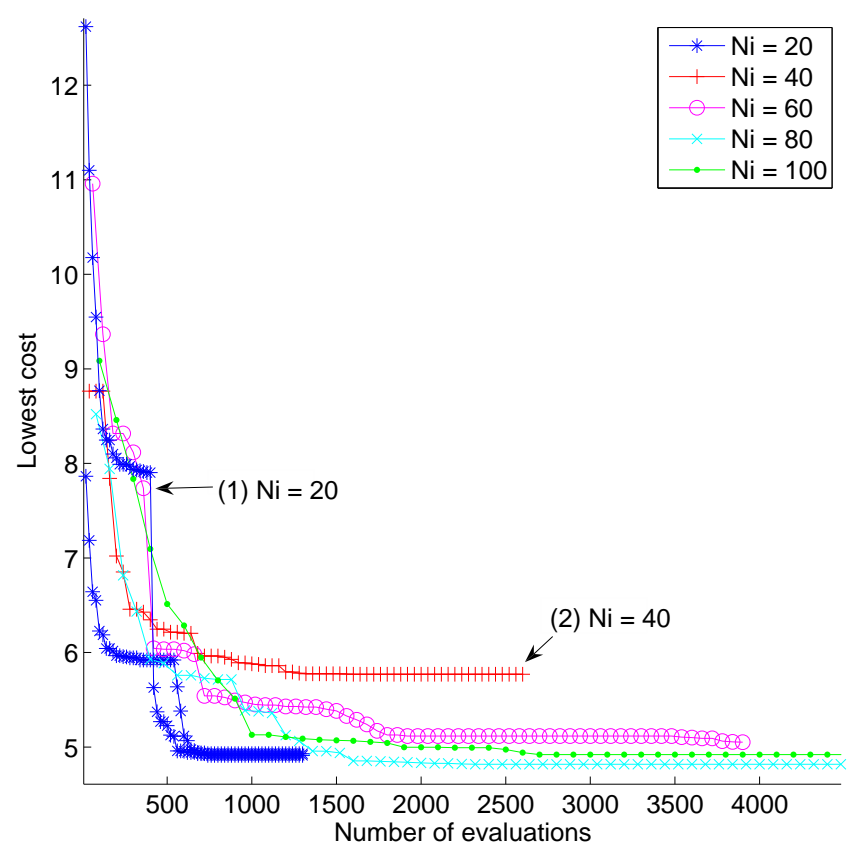

Fig. 5. Evolution of the lowest cost according to the cost function number of evaluation, for several convergence tests and with different values of $\mathrm{Ni}$.

ing to the population size. This parameter is crucial to ensure as far as possible to find the global minima of the cost function. In this perspective, a test with multiple realisations have been carried out to study the convergence of EA1 (the parameter space of EA2 is smaller than the parameters space of EA1 so it can be naturally supposed that EA2 will correctly converge for the same condition as EA1) for different numbers $\mathrm{Ni}$ of individuals $(N i=\{20,40,60,80,100\})$. For each of these numbers, the optimisation is run 6 times. Figure 5 a) presents the evolution of the cost function of the best individuals over the number of evaluation and for several tests. It can be observed that there is a global convergence around a cost of 5, whatever the number of individuals in the population. However looking more deeply on the tests performed with $\mathrm{Ni}$ $=20$, it is clear that the convergence is achieved only with the occurrence of a few good mutations or crossovers: the curves are not as smooth as with more individuals and the lowest score is sometimes decreasing abruptly (curve noted (1)) or remains at a high value. This effect is less visible with more individuals $(\mathrm{Ni}=40$ to 100$)$. It can be observed that with $\mathrm{Ni}=$ 20 or $\mathrm{Ni}=40$, the minimum of the cost function is not always reached (for example, the case noted (2) figure 5). From this experiment, $\mathrm{Ni}=60$ represents a good compromise between the number of evaluation of the cost function and the minimum of this cost.

\section{B. Delineation results}

Jane [22] has proposed a general framework to analyse the results of delineator algorithms evaluated on a given database, such as the QTDB. Our results are issued from the same framework and compared to those achieved by [2] and [21] (a low-pass-differentiator-based algorithm). Mean errors and standard deviations are weighted by the number of beats per record, and averaged over all the records of the test set (equations 1 and 2). As we disposed of two channels and cardiologists used a combination of both to detect only one position, we chose, for each point, the channel with the lowest error. The detection error probabilities $\left(\right.$ Perr $\left._{p}\right)$ are derived from the sensitivity and specificity, computed as in [2]. All these criteria are evaluated for all indicators. Fig. 6 shows the distributions of our results over the different test sets. The cross $(+)$ and the stars $(*)$ are respectively the results from [2] and [21], as presented in these references.

A few comments can be made for each criterion:

- For the mean deviation, the median of the results are closer to 0 than the two other methods for the points Pon, Ppeak and QRSon. It is also better than [2] for Poff and better than [21] for QRSoff, Tpeak and Toff.

- For the standard deviation, the median is lower for Pon, Peak, Poff, QRSon and Tpeak, higher than [2] for Toff and higher than both [2] and [21] for QRSoff.

- The detection error probability is lower for the $\mathrm{P}$ wave but not for the $\mathrm{T}$ wave.

The behaviour of the detection error probability is more difficult to analyse than the other criteria because all the beats automatically segmented have not been manually annotated, leading to a biased measure of the specificity. To check that the obtained detection error probability is well optimized, an additional test has been performed: Receiver Operating Characteristic (ROC) curves for $\mathrm{P}$ and $\mathrm{T}$ wave detection are plotted for a test set and it is checked if the points that minimize the error probability on the ROC curves, are close to the points obtained by the optimization process, on the corresponding training set (the points given by the optimized parameters $\epsilon_{P}$ or $\left.\epsilon_{T}\right)$.

Fig. 7 shows the ROC curve for $\mathrm{P}$ wave detection performance, varying according to the $\epsilon_{P}$ parameter (from 0 to 0.43 with a step of 0.01 ). The point minimizing the probability of detection is found to be $\epsilon_{P}=0.17$ with the ROC curve whereas this parameter have been set to $\epsilon_{P}=0.15$ during the optimization process.

Other indicators of the quality of the optimization are the scores and partial scores, computed in the cost function, for all the 13 test sets generated. These scores are presented in table III. They correspond to the ratio of our three criteria (mean error, standard deviation error and detection probability error), which are lower than one if better results are achieved compared to the best results published in [2] and [21] (higher than one otherwise), and which are summed over the 7 different indicators $p$.

Several observations can be drawn from this table:

- a majority of partial costs are below 7, thus indicating that some improvements have been achieved. The mean cost over the populations is 19.38, it notices a global improvement of $7.7 \%$ over the best results found in [2] and [21].

- there is not particularly one criterion that has been optimized at the expense of the others: none of them are always below or above 7 for all the tests. This argues for 

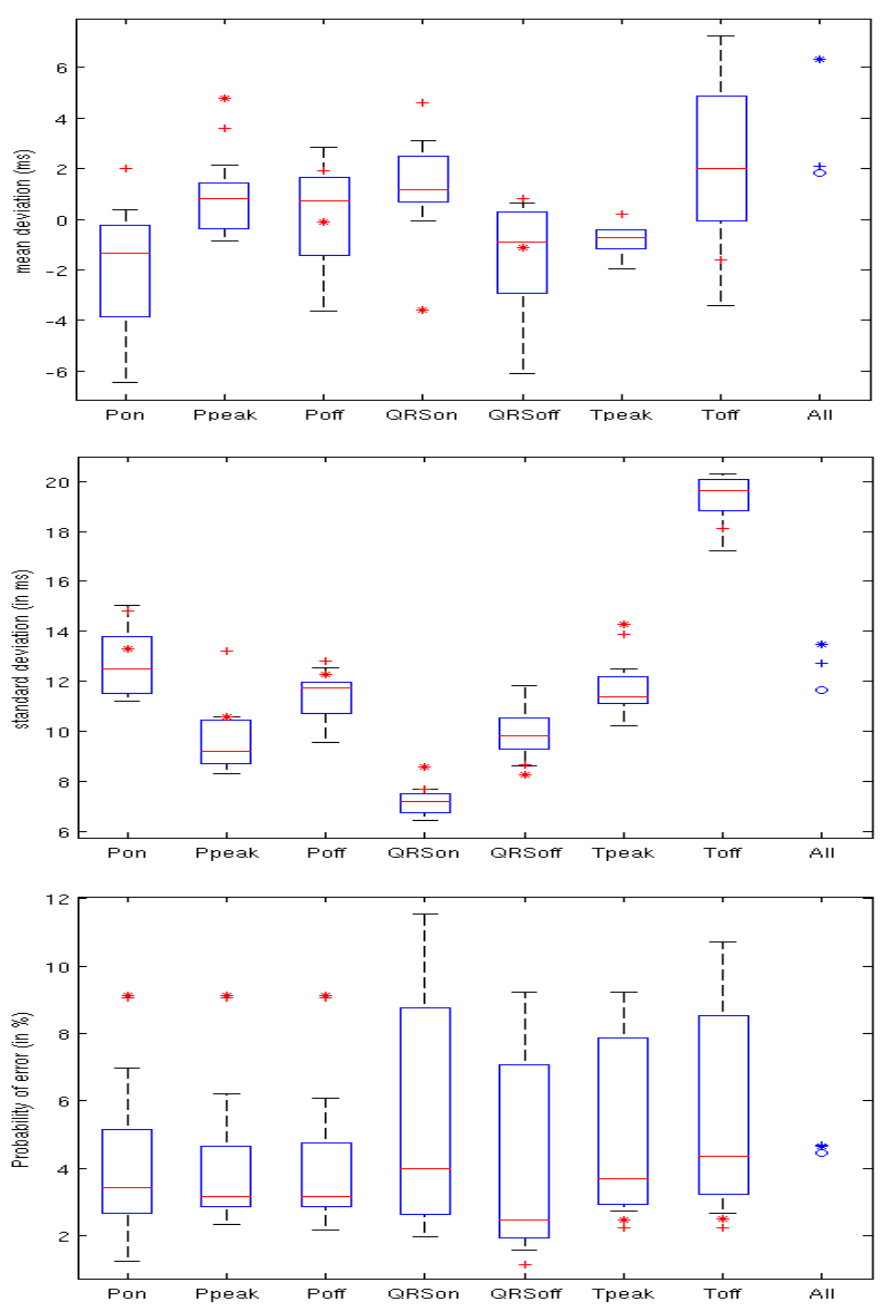

Fig. 6. Boxplots of the 3 criteria evaluated (mean, standard deviation and Perr) for all the indicators.

\begin{tabular}{|c|c|c|c|c|c|c|c|c|c|c|c|c|c|c|}
\hline populations & $\mathrm{T} 1$ & $\mathrm{~T} 2$ & $\mathrm{~T} 3$ & $\mathrm{~T} 4$ & $\mathrm{~T} 5$ & $\mathrm{~T} 6$ & $\mathrm{~T} 7$ & $\mathrm{~T} 8$ & $\mathrm{~T} 9$ & $\mathrm{~T} 10$ & $\mathrm{~T} 11$ & $\mathrm{~T} 12$ & $\mathrm{~T} 13$ & $\overline{T_{i}} \pm \sigma\left(T_{i}\right)$ \\
\hline $\bar{\varepsilon}$ & 2.66 & 4.05 & 7.7 & 3.72 & 5.75 & 7.04 & 5.97 & 6.56 & 5.2 & 4.07 & 3.42 & 4.02 & 5.22 & $5.03 \pm 1.52$ \\
\hline$\sigma$ & 6.69 & 6.59 & 6.71 & 6.20 & 6.65 & 6.7 & 5.97 & 7.33 & 6.82 & 7.21 & 6.73 & 7.25 & 6.78 & $6.74 \pm 0.38$ \\
\hline Perr & 2.88 & 5.95 & 12.69 & 5.26 & 14.14 & 6.92 & 5.49 & 6.32 & 8.81 & 13 & 2.98 & 4.5 & 10.92 & $7.68 \pm 3.85$ \\
\hline cost O & 12.23 & 16.60 & 27.11 & 15.19 & 26.52 & 18.66 & 18.50 & 20.20 & 20.83 & 24.27 & 13.13 & 15.78 & 22.92 & $19.38 \pm 4.83$ \\
\hline
\end{tabular}

TABLE III

COST FOR THE DIFFERENT TEST POPULATIONS.

the fact that improvements have been achieved globally, on all the criteria.

- the standard deviation computed over all the test sets is high.

The latter point has to be examined in more detail: it can be either due to a bad tuning of the parameters for some specific learning populations or due to some test populations that contain ECG records which are hardly delineated. Tables IV and $\mathrm{V}$ give an answer to this point.

When the best or worst optimal parameter sets $\left(O P_{L 1}\right.$ and $O P_{L 3}$ respectively) are applied to delineate all the test populations, a very high variance is observed table IV: 4.35 and 5.49 respectively. In the other hand, the best or worst test populations (T1 and T3) provide a smaller variance (1.98 and 1.85 in table $\mathrm{V}$ ) when the different parameters are applied to delineate them. From these two experiments, the high standard deviation in table III can mainly be explained by the fact that few records, those presenting some very specific pathologies, can lead to a high cost result for the test sets that contain these records (for example T3 and T5). The learning stage has less effect on this high variance. However, over the different test sets, it is possible to observe that results have been improved.

An additional test was also performed concerning the verification step introduced in the algorithm of Martinez (see section II-A). By omitting this verification step, the mean score is evaluated to 21.9 instead of 19.38 (table III). This 


\begin{tabular}{|c|c|c|c|c|c|c|c|c|c|c|c|c|c|c|}
\hline & $\mathrm{T} 1$ & $\mathrm{~T} 2$ & T3 & $\mathrm{T} 4$ & T5 & T6 & $\mathrm{T} 7$ & $\mathrm{~T} 8$ & T9 & T10 & T11 & T12 & T13 & $\overline{T_{i}} \pm \sigma\left(T_{i}\right)$ \\
\hline Best parameters $\left(O P_{L 1}\right)$ & 12.2 & 14.3 & 22.5 & 15.9 & 25.6 & 14.4 & 15.9 & 17.1 & 20.8 & 22.3 & 14.6 & 14.0 & 23.0 & $17.9 \pm 4.35$ \\
\hline Worst parameters $\left(O P_{L 3}\right)$ & 12.6 & 17.5 & 27.1 & 15.9 & 26.9 & 18.2 & 15.9 & 20.8 & 20.0 & 26.2 & 16.8 & 14.1 & 24.3 & $19.7 \pm 5.49$ \\
\hline
\end{tabular}

TABLE IV

COSTS OBTAINED WHEN APPLYING THE BEST AND WORST OPTIMIZED PARAMETERS TO ALL THE TEST POPULATIONS.

\begin{tabular}{|c|c|c|c|c|c|c|c|c|c|c|c|c|c|c|}
\hline$O P$ & $L 1$ & $L 2$ & $L 3$ & $L 4$ & $L 5$ & $L 6$ & $L 7$ & $L 8$ & $L 9$ & $L 10$ & $L 11$ & $L 12$ & $L 13$ & $\overline{O P_{L i}} \pm \sigma\left(O P_{L i}\right)$ \\
\hline Best test pop (T1) & 12.2 & 14.3 & 12.6 & 12.4 & 14.3 & 13.1 & 15.4 & 19.3 & 14.2 & 13.6 & 11.5 & 11.7 & 13.2 & $13.69 \pm 2.03$ \\
\hline Worst test pop (T3) & 22.5 & 23.5 & 27.1 & 26.2 & 25.9 & 29.7 & 21.2 & 23.1 & 19.0 & 23.2 & 20.8 & 22.7 & 20.1 & $23.45 \pm 3.04$ \\
\hline
\end{tabular}

TABLE V

COSTS OBTAINED WHEN DELINEATING THE BEST AND WORST TEST POPULATIONS WITH ALL THE OPTIMIZED PARAMETERS.

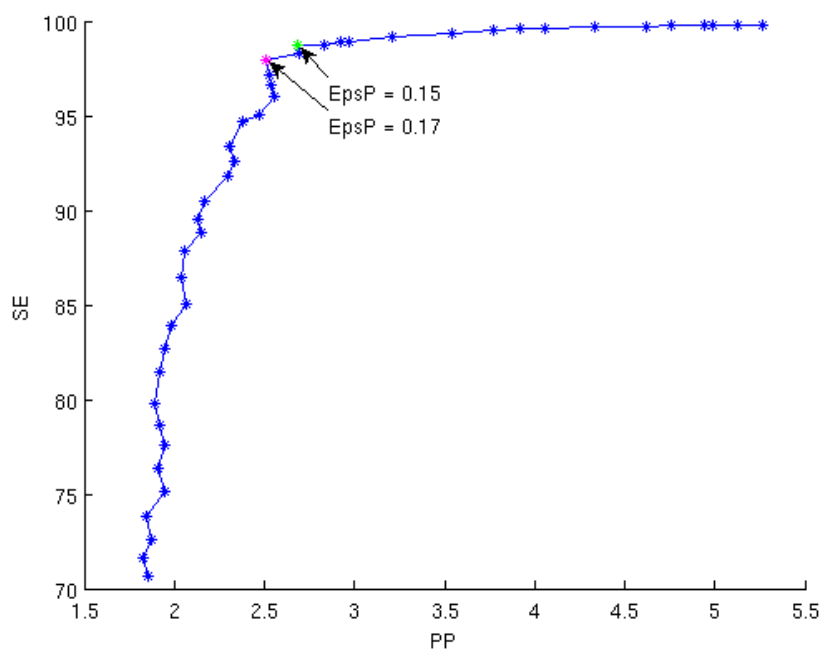

Fig. 7. ROC curve for detection performance of the $\mathrm{P}$ wave according to the $\epsilon_{P}$ parameter.

last test denotes a less accurate segmentation in this case. As an example, the $\mathrm{T}$ wave offset segmentation is affected by a standard deviation of $19.1 \mathrm{~ms}$ which is reduced to $17.6 \mathrm{~ms}$ with our improvement.

\section{CONCLUSION}

In this work we have proposed a method to obtain an optimal set of parameters for a given segmentation algorithm. The interest of this method has been demonstrated by using a modified version of the algorithm proposed by Martinez et al. It has been shown that without any a priori information on the optimal parameter distribution, the proposed optimization process provides interesting combinations of parameters to minimize a cost function and improve the global performance of the segmentation algorithm. Indeed, better results are obtained for most of the points evaluated compared to other WTbased algorithms. The optimal parameter values obtained from this method with the QTDB have been presented and their sensitivity has been analysed. These results can be useful for the implementation of WT-based ECG segmentation methods, such as those cited in this paper. It is also important to recall that the proposed methodology is generic and could be used for other biomedical applications such as detection, beat classification or epileptic spikes delineation in EEG records, but also in others fields.

Although the results reported here are interesting, several improvements can be carried out. The first one could be to perform an insight study of the individuals remaining in the final population of the EA. This could be very useful for the development of the segmentation procedure. For example, if the chromosomes tend to divide into two distinct populations, differentiated by only one or two parameters, it would mean that there exists two different local minima of the cost function with similar values. The set of parameters leading to one of these minima may produce a good segmentation for only one type of beat morphology; the other set would be accurate for the remaining morphologies. Thus, according to the parameters in question, some improvements could be made in the algorithm by introducing heuristic rules that would permit to identify these two distinct morphologies. The second improvement could be to transform some of our fixed parameters to adaptive parameters. The new parameters could be adjusted depending on the current noise level, the heart rate or the observed wave morphology. Finally, although records of the QTDB offer a wide panel of beat morphologies, and thus should avoid generalization problems, it would be interesting to evaluate this algorithm on other standard ECG databases.

\section{REFERENCES}

[1] C. Li, C. Zheng, and C. Tai, "Detection of ecg characteristic points using wavelet transform," IEEE Trans on Biomed Eng, vol. 41, pp. 2128, 1995.

[2] J. P. Martínez, R. Almeida, S. Olmos, A. P. Rocha, and P. Laguna, "A wavelet-based ECG delineator: evaluation on standard databases." IEEE Trans Biomed Eng, vol. 51, no. 4, pp. 570-581, Apr 2004.

[3] N. Sivannarayana and D. C. Reddy, "Genetic algorithms + data structures = evolution programs," Med Eng Phys, vol. 21, no. 3, pp. 167-174, Apr 1999.

[4] J. S. Sahambi, S. N. Tandon, and R. K. Bhatt, "Wavelet based STsegment analysis." Med Biol Eng Comput, vol. 36, no. 5, pp. 568-572, Sep 1998.

[5] M. Bahoura, M. Hassani, and M. Hubin, "DSP implementation of wavelet transform for real time ECG wave forms detection and heart rate analysis." Comput Methods Programs Biomed, vol. 52, no. 1, pp. 35-44, Jan 1997.

[6] P. Laguna, R. Mark, A. Goldberger, and G. Moody, "A database for evaluation of algorithms for measurement of QT and other waveform intervals in the ECG," Computers in Cardiology, vol. 24, pp. 673-676, 1997.

[7] J. A. Vila, Y. Gang, J. M. R. Presedo, M. Fernndez-Delgado, S. Barro, and M. Malik, "A new approach for TU complex characterization." IEEE Trans Biomed Eng, vol. 47, no. 6, pp. 764-772, Jun 2000. 
[8] E. Soria-Olivas, M. Martínez-Sober, J. Calpe-Maravilla, J. F. GuerreroMartínez, J. Chorro-Gascó, and J. Espí-López, "Application of adaptive signal processing for determining the limits of $\mathrm{P}$ and $\mathrm{T}$ waves in an ECG.” IEEE Trans Biomed Eng, vol. 45, no. 8, pp. 1077-1080, Aug 1998.

[9] H. Vullings, M. Verhaegen, and H. Verbruggen, "Automated ecg segmentation with dynamic time warping," Proc. 20th Ann. Int. Conf. IEEE Engineering in Medecine and Biology Soc. Hong Kong, pp. 163-166, 1998.

[10] V. Shusterman, S. I. Shah, A. Beigel, and K. P. Anderson, "Enhancing the precision of ECG baseline correction: selective filtering and removal of residual error." Comput Biomed Res, vol. 33, no. 2, pp. 144-160, Apr 2000.

[11] J. Pan and W. J. Tompkins, "A real-time QRS detection algorithm." IEEE Trans Biomed Eng, vol. 32, no. 3, pp. 230-236, Mar 1985.

[12] M. Holschneider, R. Kronland-Martinet, M. Morlet, and P. Tchamitchian, "A real-time algorithm for signal analysiswith the help of the wavelet transform," in Wavelets, Time-Frequency Methods and Phase Space, Springer-Verlag, Ed., Berlin, 1989, pp. 289-297.

[13] A. Smrdel and F. Jager, "Automated detection of transient ST-segment episodes in 24h electrocardiograms," Med Biol Eng Comput, vol. 42, no. 3, pp. 303-311, May 2004

[14] P. Laguna, N. V. Thakor, P. Caminal, R. Jané, H. Yoon, A. Bayés de Luna, A. V. Marti, and J. Guindo, "New algorithm for qt interval analysis in 24-hour holter ecg: performance and applications," in Medical and Biological Engineering and Computing, S. B. . Heidelberg, Ed., vol. 28, no. 1, January 1990 , pp. 67-73.

[15] Z. Michalewicz, Genetic Algorithms + Data Structures $=$ Evolution Programs. Springer, Berlin and Heidelberg, 3rd edition, 1996.

[16] A. I. Hernández, G. Carrault, F. Mora, and A. Bardou, "Model-based interpretation of cardiac beats by evolutionary algorithms: signal and model interaction." Artif Intell Med, vol. 26, no. 3, pp. 211-235, Nov 2002.

[17] R. E. West, E. D. Schutter, and G. Wilcox, "'using evolutionary algorithms to search for control parameters in a nonlinear partial differential equation"," University of Minnesota Supercomputer Institute Research, Report UMSI 97/61, Tech. Rep., April 1998.

[18] F. de Toro, E.Ros, S.Mota, and J.Ortega, "Evolutionary algorithms for multiobjective and multimodal optimisation of diagnostic schemes," IEEE Transactions on Biomedical Engineering, vol. 53, no. 2, pp. 178189, 2006.

[19] V. Khare1, X. Yao1, and K. Deb, "Performance scaling of multiobjective evolutionary algorithms," in Lecture Notes in Computer Science, Evolutionary Multi-Criterion Optimization: Second International Conference. Springer Berlin / Heidelberg, February 2004, p. 72.

[20] T. Back and M. Schutz, "Intelligent mutation rate control in canonical genetic algorithms," Proc of the International Sympoisum on Methodologies for Intelligent Systems, pp. 158-167, 1996.

[21] P. Laguna, R. Jané, and P. Caminal, "Automatic detection of wave boundaries in multilead ECG signals: validation with the CSE database." Comput Biomed Res, vol. 27, no. 1, pp. 45-60, Feb 1994.

[22] R. Jané, A. Blasi, J. Garcia, and P. Laguna, "Evaluation of an automatic detector of waveforms limits in holter ECG with the QT database," Computers in Cardiology, vol. 29, pp. 295-298, 1997.

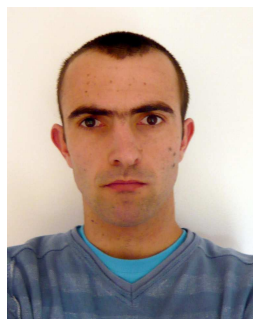

Jerome Dumont Jerome Dumont received the Master of Engineering degree in electronic and electrical engineering from the University of Strathclyde, in 2004, in Glasgow. He is working since 2004 as a $\mathrm{PhD}$ student in the Signal and Image Processing Laboratory (LTSI) of the University of Rennes 1. His research interests are in biomedicad digital signal processing and spatio-temporal data mining.

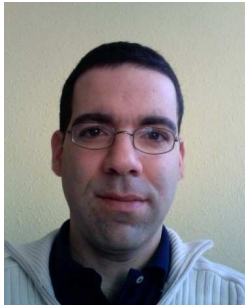

Alfredo I. Hernández Alfredo I. Hernández received the M.S. degree in electronic engineering (biomedical option) from the Simón Bolívar University in 1996 in Caracas, Venezuela and his Ph.D. degree in signal processing and telecommunications in 2000 from the University of Rennes 1, France. He is working since 2001 as a full-time researcher at the French National Institute of Health and Medical Research (INSERM) with the Signal and Image Processing Laboratory (LTSI) of the University of Rennes 1 . His research interests are in biomedical digital signal processing and model-based biosignal interpretation.

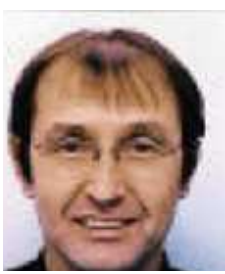

Guy Carrault Guy Carrault received his $\mathrm{PhD}$ in 1987 in Signal processing and telecommunications from the Universit de Rennes 1. He is working in the Signal and Image Processing laboratory of Universit de Rennes 1 since 1984 . He is currently professor at the Institut Universitaire de Technologie de Rennes. His research interests include detection and analysis of electrophysical signals by means of nonstationnary and statistical processing methods, as well as intelligent instrumentation design. 